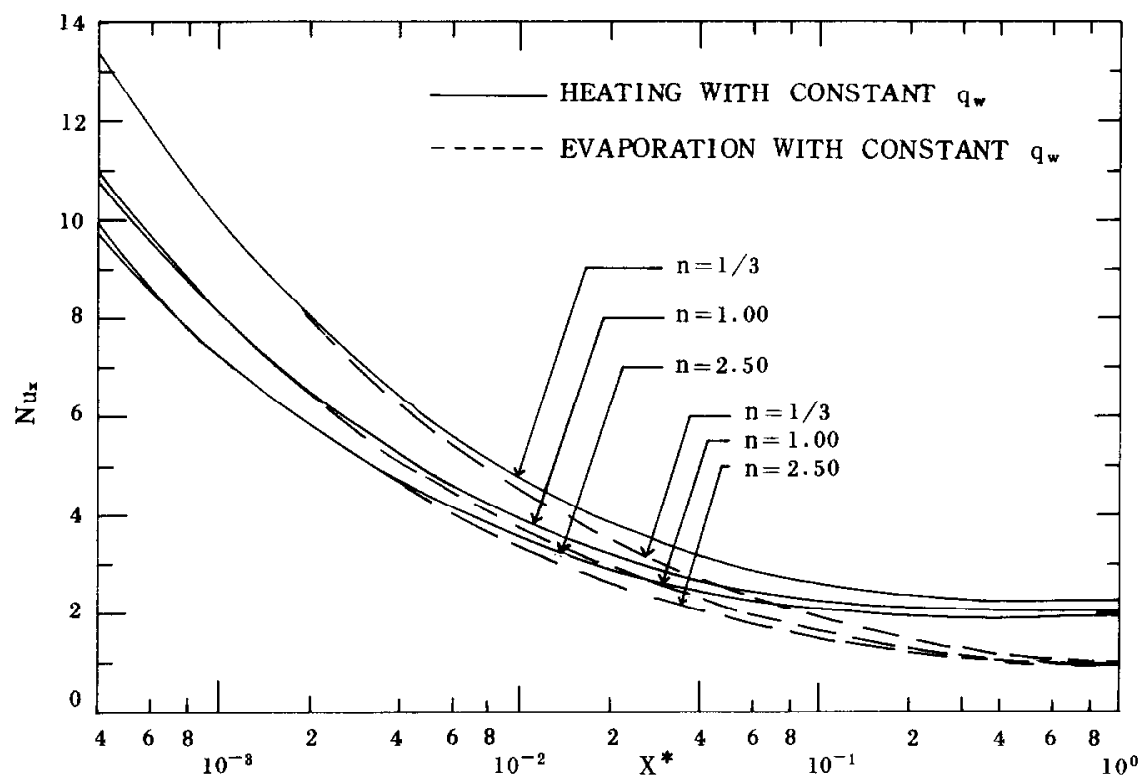

FIG. 2. Comparison of local Nusselt number for heating and evaporation with constant wall heat flux.

\section{REFERENCES}

1. V. N. Murthy and P. K. Sarma, Heat transfer to nonNewtonian laminar falling liquid films with smooth wave free gas-liquid interface, Ini. J. Multiphase Flow 4, 413425 (1978).

2. A. Stucheli and F. Widmer, Nicht-isotherme rieselfilmstromung einer hochviskosen flussigkeit mit stark temperaturabhangiger viskositat, Warme- $u$. Stoffubertr. 11, 91-101 (1978).

3. S. M. Yih and P. G. Huang, Solid dissolution with or without chemical reaction in laminar non-Newtonian falling liquid films, J. Chin. Inst. chem. Engrs 11, 71-84 (1980).
4. S. M. Yih and C. H. Chen, Heat transfer in the thermal entrance region of a turbulent falling liquid film undergoing heating or evaporation, Proc. 7th Int. Heat Transfer Conference, Munich, Vol. 3, pp. 125-130 (1982).

5. S. M. Yih and J. L. Liu, Prediction of heat transfer in turbulent falling liquid films with or without interfacial shear, A.I.Ch.E. Jl 29, 903-909 (1983).

6. R. A. Mashelkar and V. V. Chavan, Solid dissolution in falling films of non-Newtonian liquids, J. chem. Engng Japan 6, 160-167 (1973).

7. S. M. Yih and R. C. Seagrave, Mass transfer in laminar falling liquid films with accompanying heat transfer and interfacial shear, Int. J. Heat Mass Transfer 23, 749-758 (1980).

\title{
Some aspects of enhanced heat diffusion in fluids by oscillation
}

\author{
M. Kaviany \\ Department of Mechanical Engineering and Applied Mechanics, University of Michigan, \\ Ann Arbor, MI 48109, U.S.A.
}

(Received 10 December 1985 and in final form 11 March 1986)

\section{INTRODUCTION}

THE SUBJECT of enhanced heat transfer (passive or active) can be divided into two areas : (i) improvement of heat transfer rates at solid surface-fluid interfaces; and (ii) improvement in transport capabilities of the conducting fluid. In the former case it has been shown theoretically and experimentally that the oscillation of the surface can increase the heat transfer rate, and various geometries and techniques have been explored [1-3]. In the latter case a variety of methods have been considered, including wicked or gravitydriven heat pipes and packed-bed heat pipes [4]. Recently the laminar oscillation of an otherwise stationary fluid has also been studied [5-11]. The oscillation technique, which is the subject of this study, is based on the periodic longitudinal convective-lateral diffusive thermal energy transport in the presence of a longitudinal temperature gradient. This can result in a very significant increase in the longitudinal transport capability of the fluid.

This idea was initially applied to the enhancement of longitudinal oxygen dispersion in pulsating flows in pulmonary systems $[5,6,8]$ and has recently been applied to axial transport of thermal energy [9-11].

The available studies of this phenomenon, which are based on the application of the conservation equations to periodic laminar flows, have led to the prediction of the longitudinal heat transfer rates $[10,11]$. Closed-form solutions have been found for one-dimensional and linear velocity and temperature fields. Experimentally [9], enhanced heat transfer rates have been found using water as the fluid, 1-mm-ID capillary tubes, oscillation frequencies between 2 and $8 \mathrm{~Hz}$ and tidal displacements (i.e. the average particle dis- 


\section{NOMENCLATURE}

$a \quad$ half channel width [m]

$b \quad$ half wall thickness [m]

$c \quad$ specific heat $\left[\mathrm{J} \mathrm{kg}^{-1} \mathrm{~K}^{-1}\right]$

$E_{c} \quad$ Eckert number, $\left(a^{2} \omega^{2}\right) /\left(c_{\mathrm{f}} \Delta T\right)$

$g$ spatial component of the oscillating

temperature [m]

$k$ thermal conductivity $\left[\mathrm{W} \mathrm{m} \mathrm{m}^{-1} \mathrm{~K}^{-1}\right]$

$P \quad$ pressure $\left[\mathrm{N} \mathrm{m}^{-2}\right]$

$P_{x \max }$ absolute value of maximum pressure

gradicnt $\left[\mathrm{N} \mathrm{m}^{-3}\right]$

$P e^{\prime} \quad$ Péclet number, $u_{\mathrm{c}} a / \kappa_{\mathrm{r}}$

$\operatorname{Pr} \quad$ Prandtl number, $v / \kappa$

$q \quad$ heat flux [W $\left.\mathrm{m}^{-2}\right]$

$R^{\prime} \quad$ Reynolds number, $u_{c} a / v$

$u \quad$ velocity $\left[\mathrm{m} \mathrm{s}^{-1}\right]$

$u_{\mathrm{c}} \quad$ characteristic velocity, $p_{x, \max } /\left(\rho_{\mathrm{f}} a \omega^{2}\right)\left[\mathrm{m} \mathrm{s}^{-1}\right]$

$T$ temperature [K]

$\bar{T}_{x} \quad$ time-averaged temperature gradient $\left[\mathrm{K} \mathrm{m}^{-1}\right]$

$t$ time [s]

$x, y \quad$ coordinate axes $[\mathrm{m}]$

\section{Greek symbols}

$\alpha \quad$ Womersley or Stokes number, $a \omega^{1 / 2} / v^{1 / 2}$ $\theta$

oscillatory component of the temperature,

$g \mathrm{e}^{\mathrm{i} t}[\mathrm{~m}]$

frequency $\left[\mathrm{rad} \mathrm{s}^{-1}\right.$ ]

$\kappa$ thermal diffusivity $\left[\mathrm{m}^{2} \mathrm{~s}^{-1}\right]$

\& $\quad(a+b) / a$

$\rho$ density $\left[\mathrm{kg} \mathrm{m}^{-3}\right]$

$\Phi$ viscous dissipation $\left[\mathrm{m}^{2} \mathrm{~s}^{-2}\right]$

$v \quad$ kinematic viscosity $\left[\mathrm{m}^{2} \mathrm{~s}^{-1}\right]$

$\delta \quad$ Stokes viscous sublayer thickness $(2 v / \omega)^{1 / 2}[\mathrm{~m}]$

o $\quad \kappa_{\mathrm{f}} / \kappa_{\mathrm{s}}$

$\mu \quad k_{\mathrm{i}} / k$

$\Delta T \quad T_{\mathrm{h}}-T_{\mathrm{c}}[\mathrm{K}]$.

Superscripts

* time averaged

dimensional.

Subscripts

c cold

e effective

f fluid

0 fundamental

s solid

max peak

h hot. placement over half of a cycle) of between 2 and $12.5 \mathrm{~cm}$ However, the nature of the experiment did not allow for a steady-state behavior.

The concept can be applied to the transport of large heat fluxes between two reservoirs without allowing any convective mixing of the fluids contained in each reservoir.

In this study the analysis of ref. [11] is extended to include the effects of: (i) viscous dissipation, (ii) the presence of harmonics other than fundamental harmonics, (iii) channel spacing, and (iv) wall thickness. Conditions for the optimum performance are also discussed

\section{ANALYSIS}

The analysis is based on the available results given in refs. $[5,11]$. These are slightly extended and are repeated here for completeness. For quantities which appear in both dimensional and nondimensional forms, asterisks are used to indicate that they are dimensional.

The length scale of the problem can be taken as half of the channel width, $a$. The time scales are associated with the diffusion of heat in the fluid and solid, the penetration of wall shear stress, and the period of oscillation. These are $a^{2} / \kappa_{\mathrm{f}}, a^{2} / \kappa_{\mathrm{s}}, a^{2} / v$ and $1 / \omega$, respectively. Here we choose $1 / \omega$ as the time scale, and therefore $a \omega$ will be the velocity scale and $\rho_{\mathrm{f}} a^{2} \omega^{2}$ will be the pressure scale. The temperature is nondimensionalized by using $\Delta T^{*}=T_{h}^{*}-T_{c}^{*}$. Based on these scales, the dimensionless forms of the conservation equations are

$$
\begin{gathered}
\nabla \cdot \mathbf{u}=0 \\
\mathbf{u}_{\mathrm{t}}+(\mathbf{u} \cdot \mathrm{V}) \mathbf{u}=-\mathrm{V} P+\alpha^{-2} \nabla^{2} \mathbf{u} \\
T_{\mathrm{t}}+(\mathbf{u} \cdot \nabla) T=\left(\alpha^{2} P r\right)^{-1} \nabla^{2} T+u_{\mathrm{c}}^{2} E c \alpha^{-2} \boldsymbol{\Phi} \quad 0 \leqslant y \leqslant 1 \\
T_{\mathrm{l}}=\left(\sigma \alpha^{2} P r\right)^{-1} \nabla^{2} T \quad 1 \leqslant y \leqslant \varepsilon
\end{gathered}
$$

where equation (3a) describes the temperature field in the fluid and equation ( $3 b$ ) is for the confining solid. A rectangular coordinate system is chosen with $y$ in the lateral direction and $x$ in the longitudinal direction and symmetry is assumed around $y=0$. Here, $\alpha^{2}=a^{7} \omega / v$ is the ratio of the viscous penetration time scale to the oscillation time scale (Womersley or Stokes number), $\alpha^{2} P r=a^{2} \omega / \kappa_{\mathrm{f}}$ is the ratio of the thermal diffusion time scale to the oscillation time scale, and $\sigma=\kappa_{\mathrm{f}} / \kappa_{\mathrm{s}}$ is the ratio of the solid to fluid thermal diffusion time scale. Other parameters are defined in the Nomenclature. From the boundary conditions on the heat flow rate at $y=1$ (the fluid-solid interface), a parameter appears which describes the ratio of thermal conductivities, $\mu$.

The dimensionless tidal displacement is $[5,11]$

$$
\Delta x=\left|\int_{-\pi / 2}^{\pi / 2} \int u \mathrm{~d} y \mathrm{~d} t\right|
$$

and the effective thermal diffusivity is defined through

$$
-\frac{\kappa_{\mathrm{e}}}{a^{2} \omega} \bar{T}_{x}=\frac{1}{2 \pi} \int_{0}^{2 \pi} \int_{0}^{1} \operatorname{Real}(T) \operatorname{Real}(u) \mathrm{d} y \mathrm{~d} t .
$$

Then, the heat transfer rate per unit area can be defined as

$$
q=\frac{\kappa_{\mathrm{e}} \rho_{\mathrm{f}} c_{\mathrm{f}} \bar{T}_{x}^{*}}{\varepsilon}
$$

which shows that the heat transfer increases with $\rho_{\mathrm{f}} c_{\mathrm{f}}$ and $\bar{T}_{x}^{*}$ where $\bar{T}_{x}^{*}$ is the time-averaged dimensional longitudinal temperature gradient $\left(\mathrm{K} \mathrm{m}^{-1}\right)$.

For a one-dimensional (i.e. fully-developed) velocity field, the solution for velocity is the real part of $[11,13]$

$$
u=\mathrm{i} u_{\mathrm{c}}\left[1-\frac{\cosh \left(\mathrm{i}^{1 / 2} \alpha y\right)}{\cosh \left(\mathrm{i}^{1 / 2} \alpha\right)}\right] \mathrm{e}^{\mathrm{i} t}=\mathrm{i} u_{\mathrm{c}} f \mathrm{e}^{\mathrm{i} t}
$$

where $i^{1 / 2}=(1+i) / 2^{1 / 2}$ and $i=(-1)^{1 / 2}$.

The energy equations for the fluid and solid (neglecting axial conduction in the fluid and solid and for a one-dimensional velocity field) are

$$
\begin{gathered}
T_{\mathrm{t}}+\mathrm{i} u_{\mathrm{c}} \bar{T}_{x} f \mathrm{e}^{\mathrm{i} t}=\left(\alpha^{2} P r\right)^{-1} T_{y y}+u_{\mathrm{c}}^{2} E c \alpha^{-2}\left(f_{y} \mathrm{e}^{\mathrm{i} r}\right)^{2} \quad 0<y<1 \\
T_{\mathrm{t}}=\left(\sigma \alpha^{2} P r\right)^{-1} T_{y y} \quad 1<y<\varepsilon
\end{gathered}
$$

these are subject to $T_{\mathrm{f}}(1)=T_{s}(1)$ and $\mu T_{y, \mathrm{f}}=T_{y, \mathrm{~s}}$ in addition to the symmetry conditions at $y=0$ and $\varepsilon$. 


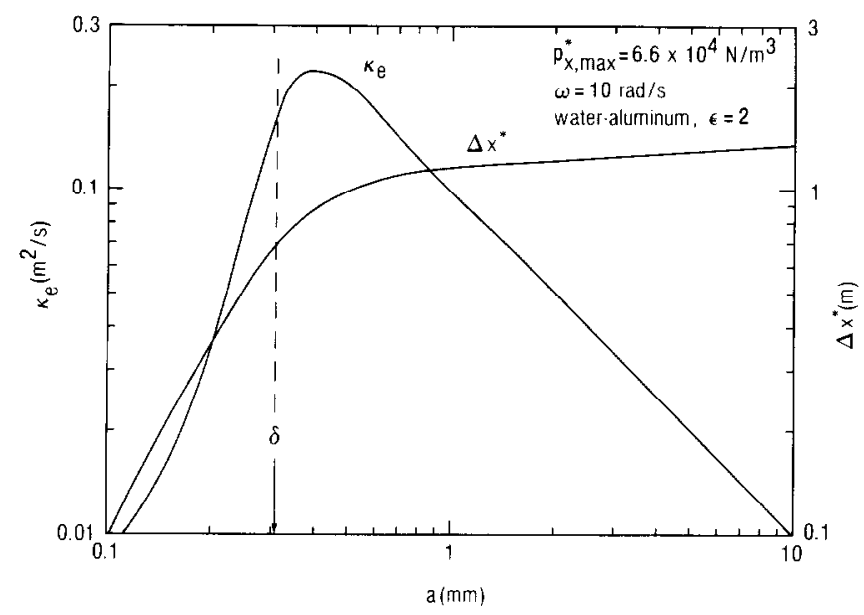

FIG. 1. The variations in the magnitude of the effective thermal diffusivity with respect to the channel width. The Stokes viscous sublayer thickness is also shown.

Now assuming that $T=\bar{T}_{x}\left(x+g \mathrm{e}^{\prime \prime}\right)=\bar{T}_{x}(x+\theta)$, where $\bar{T}_{x}$ is taken to be positive, the solution for the fluid temperature is [11]

$$
\begin{gathered}
g=c_{1} \cosh \left[(\mathrm{i} P r)^{1 / 2} \alpha y\right]+\frac{P e^{\prime}}{\alpha^{2} \operatorname{Pr}(\operatorname{Pr}-1)}-\frac{P e^{\prime}}{\alpha^{2}(\operatorname{Pr}-1)} \\
\times\left[1-\frac{\cosh \left(\mathrm{i}^{1 / 2} \alpha y\right)}{\cosh \left(\mathrm{i}^{1 / 2} \alpha\right)}\right] \quad 0 \leqslant y \leqslant 1 \\
g=c_{2} \cosh \left[(\mathrm{i} \sigma P r)^{1 / 2} \alpha(\varepsilon-1)\right] \quad 1 \leqslant y \leqslant \varepsilon
\end{gathered}
$$

where

$$
\begin{aligned}
& C_{1}=\frac{-P e\left\{\mu \operatorname{Pr}^{1 / 2} \tanh (\mathrm{i} \alpha)^{1 / 2}+\sigma^{1 / 2} \tanh \left[(\mathrm{i} \sigma P r)^{1 / 2} \alpha(\varepsilon-1)\right]\right\}}{\alpha^{2}(P r-1) \operatorname{Pr} \cosh \left[(\mathrm{i} P r)^{1 / 2} \alpha\right]\left\{\mu \tanh \left[(\mathrm{i} P r)^{1 / 2} \alpha\right]\right.} \\
& \alpha]\left\{\mu \tanh \left[(i P r)^{1 / 2} \alpha\right]\right. \\
& \left.+\sigma^{1 / 2} \tanh \left[(\mathrm{i} \sigma P r)^{1 / 2} \alpha(\varepsilon-1)\right]\right\} \\
& C_{2}=\frac{C_{1} \alpha^{2} \operatorname{Pr}(\operatorname{Pr}-1) \cosh \left[(\mathrm{i} P r)^{1 / 2} \alpha\right]+P e^{\prime}}{\alpha^{2} \operatorname{Pr}(\operatorname{Pr}-1) \cosh \left[(\mathrm{i} \sigma \operatorname{Pr})^{1 / 2} \alpha(\varepsilon-1)\right]} .
\end{aligned}
$$

The results for $\operatorname{Pr}=1$ should be obtained by taking the limit.

Then, the dimensionless tidal displacement becomes [11]

$$
\Delta x=2 u_{\mathrm{c}}\left[1-\frac{\tanh \left(\mathrm{i}^{1 / 2} \alpha\right)}{\left(\mathrm{i}^{1 / 2} \alpha\right)}\right] .
$$

The average increase in temperature due to viscous dissipation and over a cycle can be defined as

$$
\begin{aligned}
\Delta T_{\text {diss }} & \equiv \frac{1}{2 \pi} \int_{0}^{2 \pi}\left[\int_{0}^{1} T_{1} \mathrm{~d} y+\int_{1}^{c} \frac{\sigma}{\mu} T_{1} \mathrm{~d} y\right] \mathrm{d} t \\
& =\frac{1}{2 \pi} \int_{0}^{2 \pi} \int_{0}^{1} \frac{u_{\mathrm{c}}^{2} E c}{\alpha^{2}}\left(f_{y} \mathrm{e}^{\mathrm{i} t}\right)^{2} \mathrm{~d} y \mathrm{~d} t \\
& =\frac{1}{2 \pi} \int_{0}^{2 \pi} \int_{0}^{1} \frac{u_{\mathrm{c}}^{2} E c}{\alpha^{2}}\left[\mathrm{i}^{1 / 2} \frac{\sinh \left(\mathrm{i}^{1 / 2} \alpha y\right) \mathrm{e}^{\mathrm{i} t}}{\cosh \left(\mathrm{i}^{1 / 2} \alpha\right)}\right]^{2} \mathrm{~d} y \mathrm{~d} t .
\end{aligned}
$$

This is found by adding the fluid and solid energy conservation equations and then integrating the resulting equation over $0<y<\varepsilon$ and $0<t<2 \pi$, applying the boundary conditions at $y=1$ and $\varepsilon$, and then dividing by $2 \pi$.

Integration of equations (5) and (12) was done numerically using the trapezoidal rule, and progressively smaller $\Delta y$ and $\Delta t$ were used until the results no longer changed.

\section{RESULTS AND DISCUSSION}

For a given fluid and channel wall material the magnitude of effective themial conductivity depends on the peak vel- ocity $u_{c}^{*}$ and tidal displacement $\Delta x^{*}$. However, $u_{\mathrm{c}}^{*}$ and $\Delta x^{*}$ cannot be increased indefinitely due to: (a) the transition to turbulence (limitation on $u_{\mathrm{c}}^{*}$ ); and (b) the undesirable arrival of the fluid in one reservoir to the other (limitation on $\Delta x^{*}$ ). The magnitudes of $u_{c}^{*}$ and $\Delta x^{*}$ are determined by the peak pressure gradient and the frequency. The limitations on $P_{x, \max }^{*}$ and $\omega$ are mechanical. Therefore, the optimum condition is that which will maximize $\kappa_{\mathrm{e}}$ subject to these physical and mechanical constraints.

In the following, the effects of various parameters on the magnitude of $\kappa_{\mathrm{e}}$ are examined, while demonstrating the practical limitations of the constraining parameters $\left(u_{\mathrm{c}}^{*}\right.$ and $\left.\Delta x^{*}\right)$.

\subsection{Channel width}

The thermal energy storage-release process is based on heat diffusion across the channel. Therefore, the channel width cannot be made very large. Moreover, the channel width has to be larger than twice the viscous sublayer thickness, which is the arca adjacent to the wall where the effect of the wall no-slip condition is concentrated. Figure 1 shows how the effective thermal diffusivity first increases with the half channel width, $a$, and attains a maximum around $a=\delta$, and then decreases with an increase in $a$. Figure 1 also shows that for a given $P_{x, \max }^{*}$ and $\omega$, the tidal displacement increases with $a$.

\subsection{Fluid}

As given by equation (6), the longitudinal heat transfer rate increases with an increase in $\rho_{\mathrm{f}} \mathcal{c}_{\mathrm{f}}$. Also, the viscosity and thermal conductivity of the fluid influence the magnitude of the effective thermal diffusivity [through equation (10)]. Figure 2 shows the variation in $\kappa_{\mathrm{e}}$ with respect to frequency for three different fluids, namely, mercury, water and light oil. The results are for $\Delta x^{*}=1 \mathrm{~m}$, aluminum channel walls of $\varepsilon=2$, and $a=5 \times 10^{-4} \mathrm{~m}$. Note that although $\kappa_{\mathrm{e}} / \omega \Delta x^{* 2}$ reaches its maximum value for $\left(a^{2} \omega / \kappa_{\mathrm{f}}\right)=\alpha^{2} \operatorname{Pr}$ of approximately 3 , there is no maximum for $\kappa_{\mathrm{e}}$ with respect to $\omega$.

For water and light oil, there exists an intermediate range of $\omega$ for which $\kappa_{\mathrm{e}}$ does not change significantly with $\omega$.

\subsection{Channel wall}

The channel wall stores and releases thermal energy. The wall thickness and thermophysical properties should complement the properties of the fluid, among other requirements. Figure 3 shows the effect of the wall thickness on the effective thermal diffusivity. The results are for mercury as the fluid and aluminum or glass as the channel wall. Results show that for lower values of $\omega$, the magnitude of $\kappa_{\mathrm{c}}$ increases 


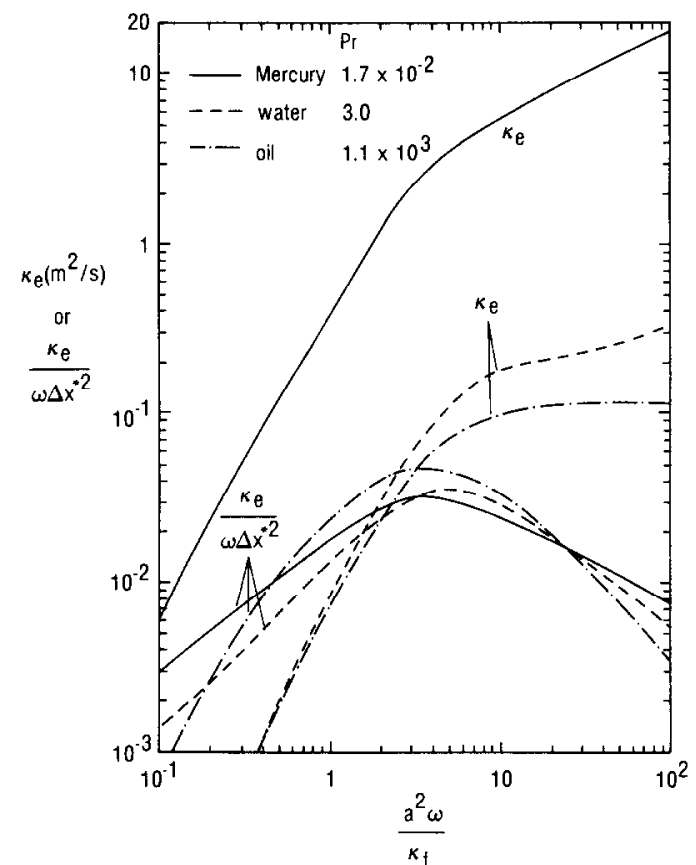

FIG. 2. The variations in the magnitude of the effective thermal diffusivity with respect to the oscillating frequency for different fluids.

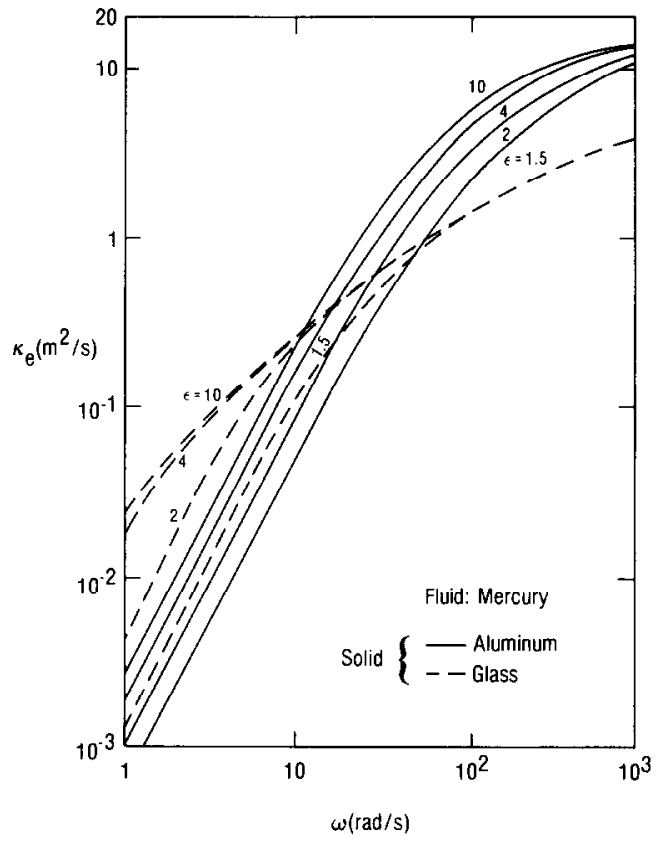

FIG. 3. The effects of wall thickness and material on the effective thermal diffusivity.

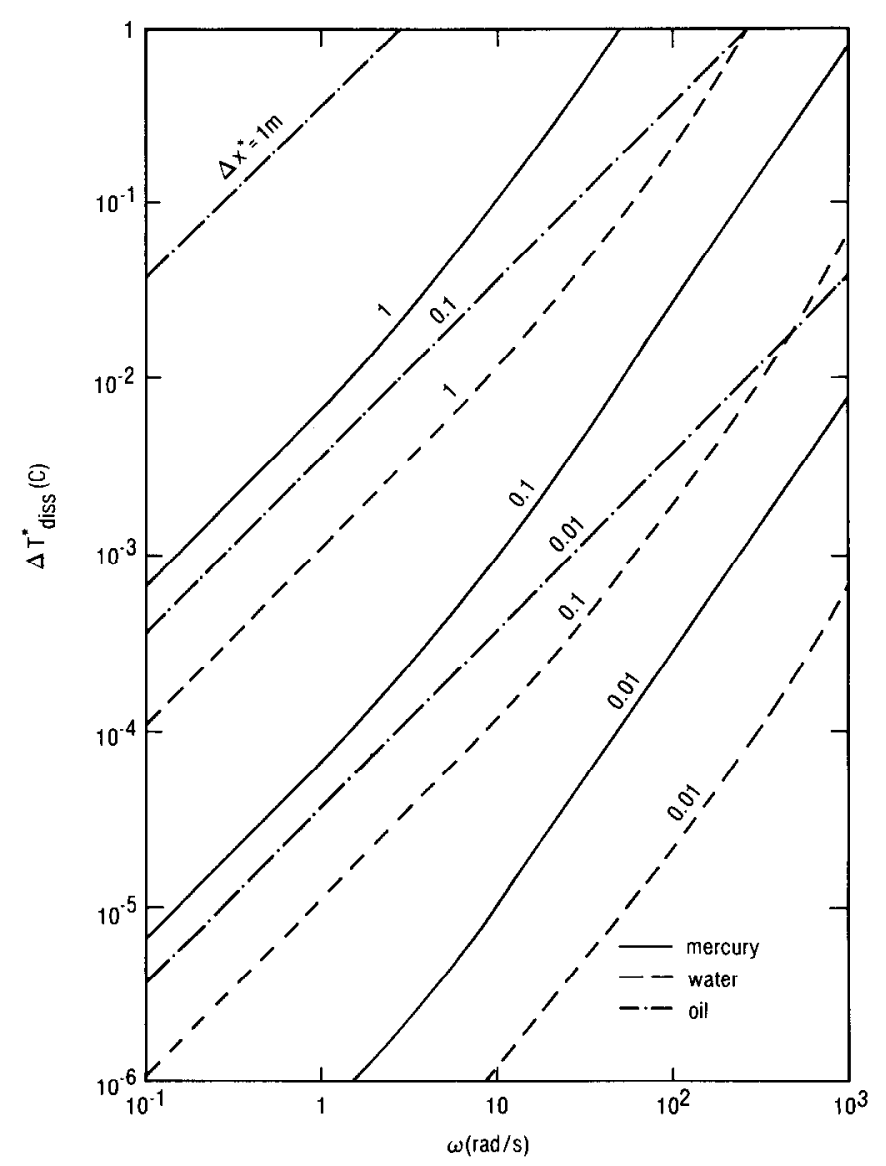

FIG. 4. The average increase in the fluid temperature over a cycle for various fluids and tidal displacements. 
with an increase in $\varepsilon$. Note that axial conduction through the solid is neglected and the longitudinal heat transfer is inversely proportional to $\varepsilon$, as given by equation (6). Therefore. for $q$ to increase with $\varepsilon, \kappa_{\mathrm{e}} / \varepsilon$ must increase with $\varepsilon$.

Figure 3 also shows that a given wall material does not optimally complement the fluid at all frequencies.

\subsection{Viscous dissipation}

Since the magnitude of $\kappa_{\mathrm{e}}$ increases with increases in $\omega$ and $u_{c}^{*}$, the viscous dissipation can become significant when large values of $\kappa_{\mathrm{e}}$ are attained. The average temperature rise during a cycle, which is obtained by integrating the energy equation, is given by equation (12). Figure 4 shows this increase in temperature as functions of frequency and $\Delta x^{*}$ for different fluids. The results are for an aluminum channel wall, $\varepsilon=2$, and $a=5 \times 10^{-4} \mathrm{~m}$. The results show that for large values of $\Delta x^{*}$ and $\omega$, even for water as the fluid, significant temperature rises can occur.

\subsection{Other harmonics}

Due to the linearity of the momentum and energy equations, the solutions for other harmonics are similar to those given in Section 2. If the magnitude of $P_{x, \max }^{*}$ is the same for all the harmonics, then for frequencies lower than the one produced by the oscillator, the tidal displacement will be larger than that for the oscillator frequency and vice versa. For lower frequencies, the magnitudes of $u_{\mathrm{c}}^{*}$ and $\Delta x^{*}$ may become so large as to either cause transition to turbulence [12] inside the channels (large $u_{c}^{*}$ ) or move the fluid from one reservoir to the other (large $\Delta x^{*}$ ). Therefore, the presence of frequencies lower than fundamental can severely alter the performance.

Acknowledgements - The financial support of the National Science Foundation through grant CBT-86-03415 is greatly appreciated.

\section{REFERENCES}

I. N. Fujita and T. Tsubouchi, Experimental study of unsteady heat transfer from a flat plate to an oscillating air flow, Heat Transfer--Japan. Res. 11, 31-43 (1982).

2. U. C. Saxena and A. D. K. Laird, Heat transfer from a cylinder oscillating in a cross-flow, $J$. Heat Transfer 100, 684689 (1978).

3. B. M. Galitseikii, A. A. Nozdrin, Yu. A. Ryzhov and E. V. Yakush, Investigation of local heat-transfer coefficient under conditions of the resonance oscillations of a gas in channels, J. Engng Phys. 31, 873-876 (1976).

4. K. S. Udell, Heat transfer in porous media considering phase change and capillarity - the heat pipe effect, Int. J. Heat Mass Transfer 28, 485-495 (1985).

5. E. J. Watson, Diffusion in oscillatory pipe flow, J. Fluid Mech. 133, 233-244 (1983).

6. U. H. Kurzweg, G. Howell and M. J. Jaeger, Enhanced dispersion in oscillatory flows, Phys. Fluids 27, 10461048 (1984).

7. E. Brocher, Oscillatory flows in ducts : a report on Euromech 73, J. Fluid Mech. 79, 113-126 (1977).

8. C. H. Joshi, R. D. Kamm, J. M. Drazen and A. S. Slutsley, An experimental study of gas exchange in laminar oscillatory flows, J. Fluid Mech. 133, 245-254 (1983).

9. U. H. Kurzweg and L. de Zhao, Heat transfer by high frequency oscillations: a new hydrodynamic technique for achieving large effective thermal conductivities, Phys. Fluids 27, 2624-2627 (1984).

10. U. H. Kurzweg, Enhanced heat conduction in fluids subjected to sinusoidal oscillations, J. Heat Transfer 107, 459-462 (1985).

11. U. H. Kurzweg, Enhanced heat conduction in oscillatory viscous flows within parallel-plate channels, J. Fluid Mech. 156, 291-300 (1985).

12. P. Grassmann and M. Tuma, Critical Reynolds number for oscillating and pulsating tube flow (in German), Thermo. Fluid Dynam. 12, 203-209 (1979).

13. V.S. Arpaci and P. S. Larson, Convection Heat Transfer, pp. 425-458. Prenticc-Hall, Englewood Cliffs, NJ (1984). 